\title{
Factores asociados a tuberculosis multidrogorresistente primaria en pacientes de Callao, Perú
}

Factors associated with primary multidrug-resistant tuberculosis in patients from Callao, Peru

\author{
Ana Cecilia Avalos-Rodríguez ${ }^{1, a}$, Fernando Jean Carlos Imán-Izquierdo, ${ }^{1, a}$, Manuel \\ André Virú-Loza ${ }^{1,2, a}$, José Cabrera-Rivero ${ }^{3, b}$, Anthony Edgar Zárate-Robles ${ }^{1, a}$, \\ Mariela Cecibet Meza-Monterrey ${ }^{1, a}$, Brenner Alberto Ñaupari-Meza, ${ }^{1, a}$, Wendy \\ Teoana Figueroa-Bravo ${ }^{1, a}$, Mirella Kristal Cajas-Socualaya ${ }^{1, a}$, Hugo Paul Castro- \\ Trujillo ${ }^{1, c}$, Talía Minerva Zapata-Susanivar ${ }^{1, a}$ \\ Facultad de Medicina San Fernando, Universidad Nacional Mayor de San Marcos, Lima, Perú. \\ ${ }^{2}$ Asociación para el Desarrollo de la Investigación Estudiantil en Ciencias de la Salud (ADIECS-UNMSM), Lima, Perú. \\ ${ }^{3}$ Responsable de la Unidad Técnica-DR, Estrategia Sanitaria Nacional de Prevención y Control de la Tuberculosis, Ministerio de Salud, Lima, Perú. \\ ${ }^{a}$ Médico Cirujanos; ${ }^{b}$ Médico Neumólogo; ${ }^{\mathrm{c}}$ Interno de Medicina.
}

\begin{abstract}
Resumen
Introducción: En el Perú, Lima y Callao constituyen regiones con riesgo alto de transmisión activa de tuberculosis. La tuberculosis multidrogorresistente se presenta en el 5,54\% de los casos nuevos de tuberculosis pulmonar (TB MDR primaria) en Lima y Callao. Sus factores de riesgo no han sido estudiados en pacientes del Callao. Objetivos: Determinar los factores de riesgo para TB MDR primaria en pacientes atendidos en centros de salud de Callao, durante los años 2009- 2010. Diseño: Estudio caso control. Lugar: Centros de Salud de Callao. Participantes: Participaron 29 pacientes con TB MDR primaria y 37 con tuberculosis sensible, según los registros del Programa de Control de Tuberculosis y las historias clínicas ubicadas en los centros de salud. Intervenciones: Toda la información fue obtenida de fuentes secundarias. En el análisis bivariado se calculó el OR de cada covariable. Para el cálculo de los OR multivariados se utilizó modelos de regresión logística. Principales medidas de resultados: Factores de riesgo para TB MDR. Resultados: En el análisis bivariado, el haber tenido contacto con pacientes TB MDR o XDR fue un factor de riesgo significativo (OR: 5,56; IC95\%: 1,05 a 29,27); Io mismo se demostró en el análisis multivariado (OR: 14,56; IC95\%: 1,52 a 139,54). Además, en el análisis multivariado, la variable edad $\geq 40$ años mostró ser un factor protector significativo (OR: 0,94; IC95\%: 0,90 a 0,99). Conclusiones: El tener contacto con pacientes TB MDR/XDR fue factor de riesgo y una edad menor a 40 años un factor protector para contraer TB MDR primaria en los pacientes del Callao estudiados.
\end{abstract}

Palabras clave: Tuberculosis, multidrogorresistente, factores de riesgo, Perú.

\section{Abstract}

Introduction: Lima and Callao are high risk areas for tuberculosis active transmission in Peru. Multidrug-resistant tuberculosis represents $5.54 \%$ of the new cases of pulmonary tuberculosis (primary MDR-TB) in Lima and Callao. Involved risk factors have not been studied in patients from Callao. Objectives: To determine risk factors for primary MDR-TB in patients attending health care facilities in Callao during 2009-2010. Design: Case-control study. Setting: Health care facilities from Callao. Participants: Twenty nine primary MDR-TB patients and 37 drug-sensitive TB patients were studied through assessment of the Tuberculosis Control Program Registries and healthcare facilities' clinical records. Interventions: Information was obtained from secondary sources. Odds-ratios were calculated by bivariate analysis. Logistic regression models were used to calculate multivariate odd-ratios. Main outcome measures: Risk factors for primary MDR-TB. Results: Prior contact with a MDR-TB or XDR-TB patient was a significant risk factor (OR: $5.56 ; 95 \% \mathrm{Cl}: 1.05-29.27)$ in bivariate analysis; the same was found in multivariate analysis (OR: 14.56; 95\% Cl: 1.52-139.54). Being 40 or more years old was a significant protective factor in multivariate analysis (OR: $0.94 ; 95 \% \mathrm{Cl}: 0.90-0.99$ ). Conclusions: Previous contact with a MDR-TB or XDR-TB patient was a risk factor, and being 40 or more years old was a protective factor for primary MDR-TB in Callao patients studied.

Keywords: Tuberculosis, multidrug- resistance, risk factors, Peru.

An Fac med. 2014;75(3):233-6 / doi: http://dx.doi.org/10.15381/anales.v75i3.9775

\section{INTRODUCCIÓN}

En el Perú, durante el año 2012 se comunicó una tasa de incidencia de 93 casos de tuberculosis (TB) por 100 000 habitantes, y en total hubo 1225 casos nuevos de tuberculosis multidrogorresistente (TB MDR) ${ }^{(1)}$. Según el Ministerio de Salud del Perú y la Or- ganización Mundial de la Salud, el 10\% de todos los casos de tuberculosis en el Perú son MDR ${ }^{(2)}$. El 82\% de casos de TB MDR y 93\% de casos de tuberculosis extremadamente resistente (TB XDR) se concentran en las zonas de Lima y Callao, constituyéndose como regiones con riesgo alto de transmisión activa de tuberculosis ${ }^{(3)}$.
La TB MDR en casos nuevos (TB MDR primaria) es definida por la Unión Internacional Contra la Tuberculosis y Enfermedades Pulmonares (IUATLD) como aquella que no ha recibido tratamiento antituberculoso previo o, en el caso de que hayan recibido terapia, esta no exceda en más de un mes ${ }^{(4)}$. 
En general, entre los casos de TB, la resistencia primaria es poco prevalente comparada con la resistencia secundaria ${ }^{(5)}$, sobretodo la multidrogorresistencia primaria. Esto último se ve reflejado en el informe de la Organización Mundial de la Salud del año 2012, el cual menciona que $5,3 \%$ de todos los casos de TB pulmonar son casos de TB MDR primaria ${ }^{(6)}$. Sin embargo, los pacientes con formas de resistencia primaria constituyen un grupo que no debe ser ignorado, pues tienen prevalencias importantes en algunas zonas. Este es el caso de la India, donde se ha estudiado en distintas zonas patrones de resistencia primaria y secundaria de TB, incluyendo TB MDR, encontrando en una de las zonas estudiadas tasas de TB MDR primaria de hasta $25 \%$ del total de casos nuevos de TB sin tratamiento previo ${ }^{(7)}$. De igual manera, en una región de Rusia se encontró una tasa de pacientes con TB MDR igual a 26\% del total de casos de TB sin tratamiento previo ${ }^{(8)}$.

Entre los factores de riesgo para desarrollar TB podemos mencionar la pobreza, la desnutrición, el hacinamiento, la reclusión o encarcelamiento, el abuso de alcohol, el consumo de drogas, el hábito de fumar, el pertenecer a determinados grupos etarios, el género, entre otros ${ }^{(9)}$. A pesar de que no existe amplia bibliografía de estos factores en TB MDR primaria, existen algunos estudios que los contemplan pero de manera aislada. El Reporte de la Vigilancia Nacional de la Resistencia a Medicamentos Antituberculosos afirma que el mayor número de casos de TB MDR primaria afectó entre los 15 y 24 años ${ }^{(2)}$. Un metaanálisis postula que el VIH puede ser un factor de riesgo para TB MDR primaria ${ }^{(10)}$, pero esto no es concluyente.

Debido a que aún no se conoce con claridad qué factores pueden predisponer a contraer TB MDR primaria, y a que en el Perú solo existen datos al respecto en la zona de Lima ${ }^{(11)}$ pero no en el Callao, consideramos necesaria y pertinente la realización de esta investigación.

\section{MÉTODOS}

El presente es un estudio caso-control el cual incluyó pacientes con TB MDR primaria diagnosticados entre enero de 2009 y diciembre de 2010. Los datos fueron recabados de los registros del Programa de Control de Tuberculosis (PCT) de la Dirección Regional de Salud del Callao (DIRESA Callao) y las historias clínicas de los centros de salud correspondientes a esa jurisdicción. Previamente, se obtuvo el permiso de acceso del Comité de Ética de la institución. De los 27 centros de salud de la región mencionada, 15 fueron incluidos en el estudio. Los centros de salud excluidos se encontraban en zonas de riesgo alto de agresión física hacia los investigadores, por presentar altos índices de violencia. Según los datos de la base de datos del Comité de Evaluación de Retratamientos Intermedios (CERI) de la Dirección Regional de Salud (DIRESA) del Callao, un total de 59 pacientes con TB MDR primaria asistían a los centros de salud de la región durante el año 2010. De estos pacientes, aquellos que acudían a los centros de salud y que no fueron incluidos en el estudio totalizaron 24 .

Se definió como caso a los pacientes diagnosticados con TB MDR primaria -según la definición de la IUATLD ${ }^{(4)}$ que vivían en la región Callao y cuyo diagnóstico había sido refrendado por el CERI de la DIRESA Callao; ellos acudían a sus centros de salud de referencia a recibir tratamiento en el momento de la realización del estudio. Se excluyó a aquellos pacientes cuyos resultados de prueba rápida MODS y agar en placa difirieron. Finalmente, se estudió a 29 pacientes con TB MDR primaria. En estos pacientes se constató, al acceder los archivos de historias clínicas de los centros de salud estudiados, que realmente habían acudido al centro de salud y cumplían con los criterios de inclusión. El grupo control incluyó pacientes TB sensibles no tratados previamente y que se encontraban recibiendo tratamiento durante el tiempo de realización del estudio en los mismos centros de salud donde fueron atendidos los pacientes con TB MDR primaria estudiados. Finalmente, se estudió 37 pacientes en el grupo control.

Registramos variables socioeconómicas (edad mayor o igual a 40 años, género masculino, grado de instrucción, ocupación y antecedente de reclusión), variables de contacto y hacinamiento (contacto con paciente TB MDR o $\mathrm{XDR}$, contacto con fallecido por TB, contacto TB intradomiciliario y número de personas por habitación en casa), variables relacionadas a comorbilidades (portador de VIH, diabetes, uso de corticoides, hospitalización previa, consumo de drogas y consumo de alcohol) $y$, finalmente, índice de masa corporal (IMC).

Se utilizó un nivel de significancia igual a 0,05 para todos los análisis. Para el análisis bivariado se calculó los odds ratios de cada variable. Para realizar nuestro análisis formulamos tres modelos de regresión múltiple. Se utilizó el software estadístico STATA 11.0

\section{RESULTADOS}

En el análisis bivariado (tabla 1), podemos observar que solamente la variable contacto con paciente TB MDR o XDR presentó un riesgo estadísticamente significativo de tener TB MDR primaria (OR: 5,56; IC95\%: 1,05 a 29,27).

Formulamos tres modelos de regresión múltiple que incluyeron las variables independientes detalladas a continuación (tabla 2).

En el modelo de regresión 1, la variable contacto con paciente TB MDR o XDR demostró mantenerse como un factor de riesgo estadísticamente significativo (OR: 14,56; IC95\%: 1,52 a 139,54) (tabla 3). En el modelo de regresión 3, la variable edad mayor o igual a 40 años mostró ser un factor protector estadísticamente significativo para tener TB MDR primaria (OR: 0,94; IC95\%: 0,90 a 0,99) (tabla 3). 


\section{DISCUSIÓN}

Si solo tomamos en consideración las investigaciones realizadas para evaluar los factores de riesgo para presentar específicamente TB MDR primaria, podemos mencionar que existen dos factores de riesgo principales identificados: estado VIH positivo ${ }^{(8)}$ y contactos intradomiciliarios con tuberculosis ${ }^{(5)}$.

Teniendo en cuenta la evidencia disponible, parece ser que la condición VIH positivo se asocia a TB MDR primaria mas no a TB MDR secundaria ${ }^{(12-15)}$. Una de las explicaciones que se han planteado para estos hallazgos es el hecho de que los pacientes que presentan VIH o TB MDR tienen más probabilidad de haber tenido estancias hospitalarias, de manera que cabe la posibilidad de que en estas estancias aumenten la probabilidad de infección de pacientes VIH positivos no tuberculosos con cepas de TB multidrogorresistentes provenientes de otros pacientes ${ }^{(10)}$. En nuestro estudio, no pudimos demostrar que el estado VIH positivo fue un factor de riesgo para tener TB MDR primaria. Lo expuesto previamente representa una explicación basada en lo que ocurre en el ambiente hospitalario. Sin embargo, en nuestro estudio hemos trabajado con pacientes ambulatorios, por lo que el presente estudio no es capaz de reflejar el efecto de la interacción entre pacientes que se da en el ambiente hospitalario. Por tanto, es necesario considerar que el fenómeno de la TB MDR primaria responde a posibles factores causales que actúan de forma distinta en el ámbito hospitalario y en el ambulatorio y, bajo este concepto, es necesario realizar en nuestro medio más investigaciones en ambos ámbitos, ya que cabe la posibilidad de que el estado VIH positivo esté asociado a TB MDR primaria en pacientes hospitalarios mas no en ambulatorios.

Encontramos que tener un contacto con TB MDR o TB XDR es un factor de riesgo estadísticamente significativo, tanto en el análisis bivariado como en el multivariado. El resto de variables de
Tabla 1. Análisis bivariado de las covariables estudiadas.

\begin{tabular}{|c|c|c|c|}
\hline & $\begin{array}{c}\text { Grupo } \\
\text { TB sensible } \\
(n=37)\end{array}$ & $\begin{array}{c}\text { Grupo } \\
\text { TB MDR primaria } \\
(n=29)\end{array}$ & OR (IC95\%) \\
\hline \multicolumn{4}{|l|}{ Variables socioeconómicas } \\
\hline \multicolumn{4}{|l|}{ Edad } \\
\hline Menor de 40 & 19 & 21 & Referencia \\
\hline Mayor o igual a 40 & 18 & 8 & $0,40(0,14$ a 1,13$)$ \\
\hline \multicolumn{4}{|l|}{ Género } \\
\hline Femenino & $15(40,5 \%)$ & $12(4,1 \%)$ & Referencia \\
\hline Masculino & $22(59,5 \%)$ & $17(58,6 \%)$ & $0,96(0,36$ a 2,59$)$ \\
\hline \multicolumn{4}{|l|}{ Grado de instrucción } \\
\hline No estudia & $3(8,1 \%)$ & $1(3,4 \%)$ & Referencia \\
\hline Primaria & $4(10,8 \%)$ & $4(13,8 \%)$ & $3(0,21$ a 42,62$)$ \\
\hline Secundaria & $21(56,8 \%)$ & $14(48,3 \%)$ & $2(0,18$ a 21,22$)$ \\
\hline Superior & $9(24,3 \%)$ & $10(34,5 \%)$ & $3.33(0,29$ a 38,08$)$ \\
\hline \multicolumn{4}{|l|}{ Ocupación } \\
\hline No trabaja ni estudia & $17(45,9 \%)$ & $14(48,3 \%)$ & Referencia \\
\hline Trabaja & $15(40,5 \%)$ & $10(34,5 \%)$ & $0,81(0,27$ a 2,35$)$ \\
\hline Estudia & $5(13,5 \%)$ & $5(17,2 \%)$ & $1,21(0,29$ a 5,06$)$ \\
\hline \multicolumn{4}{|l|}{ Reclusión previa } \\
\hline No & $35(94,6 \%)$ & $27(93,1 \%)$ & Referencia \\
\hline Sí & $2(5,4 \%)$ & $2(6,9 \%)$ & $1,29(0,17$ a 9,80$)$ \\
\hline \multicolumn{4}{|c|}{ Variables de contacto y hacinamiento } \\
\hline \multicolumn{4}{|c|}{ Contacto con paciente TB MDR o XDR } \\
\hline No & $35(94,6 \%)$ & $22(75,9 \%)$ & Referencia \\
\hline Sí & $2(5,4 \%)$ & $7(24,1 \%)$ & $5,56(1,05$ a 29,27$)$ \\
\hline \multicolumn{4}{|c|}{ Contacto previo con fallecido por TB } \\
\hline No & $36(97,29 \%)$ & $26(89,65 \%)$ & Referencia \\
\hline Sí & $1(2,7 \%)$ & $2(6,9 \%)$ & $2,66(0,23$ a 30,95$)$ \\
\hline Datos faltantes & $0(0 \%)$ & $1(3,4 \%)$ & - \\
\hline \multicolumn{4}{|c|}{ Contacto TB intradomiciliario } \\
\hline No & $26(70,3 \%)$ & $19(65,5 \%)$ & Referencia \\
\hline Sí & $11(29,7 \%)$ & $9(31,0 \%)$ & $1,06(0,37$ a 3,05$)$ \\
\hline Datos faltantes & $0(0 \%)$ & $1(3,4 \%)$ & - \\
\hline \multicolumn{4}{|c|}{ Número de personas por habitación } \\
\hline Menos de 3 & $29(78,4 \%)$ & $22(75,9 \%)$ & Referencia \\
\hline 3 o más & $6(16,2 \%)$ & $7(24,1 \%)$ & $1,53(0,45$ a 5,22$)$ \\
\hline Datos faltantes & $2(5,4 \%)$ & $0(0 \%)$ & - \\
\hline \multicolumn{4}{|c|}{ Variables de antecedente de comorbilidades } \\
\hline \multicolumn{4}{|c|}{ Portador de VIH } \\
\hline No & $36(97,3 \%)$ & $28(96,6 \%)$ & Referencia \\
\hline Sí & $1(2,7 \%)$ & $1(3,4 \%)$ & $1,28(0,07$ a 21,47$)$ \\
\hline \multicolumn{4}{|l|}{ Diabetes } \\
\hline No & $36(97,3 \%)$ & $28(96,6 \%)$ & Referencia \\
\hline Sí & $1(2,7 \%)$ & $1(3,4 \%)$ & $1,28(0,07$ a 21,47$)$ \\
\hline \multicolumn{4}{|l|}{ Uso de corticoides } \\
\hline No & $30(81,1 \%)$ & $28(96,6 \%)$ & Referencia \\
\hline Sí & $7(18,9 \%)$ & $1(3,4 \%)$ & $0,15(0,01$ a 1,32$)$ \\
\hline Hospitalización previa & & & \\
\hline No & $31(83,8 \%)$ & $28(96,6 \%)$ & Referencia \\
\hline Sí & $6(16,2 \%)$ & $1(3,4 \%)$ & $0,18(0,02$ a 1,62$)$ \\
\hline Consumo de drogas & & & \\
\hline No & $33(89,2 \%)$ & $26(89,7 \%)$ & Referencia \\
\hline Sí & $2(5,4 \%)$ & $3(10,3 \%)$ & $1,9(0,29$ a 12,24$)$ \\
\hline Datos faltantes & $2(5,4 \%)$ & $0(0 \%)$ & - \\
\hline Consumo de alcohol & & & \\
\hline No & $18(51,4 \%)$ & $14(50 \%)$ & Referencia \\
\hline Sí & $17(48,6 \%)$ & $14(50 \%)$ & $1,05(0,39$ a 2,86$)$ \\
\hline Datos faltantes & 2 & 1 & - \\
\hline Categoría de IMC & & & \\
\hline Malnutrición & $4(10,8 \%)$ & $3(10,3 \%)$ & $0,97(0,19$ a 4,93$)$ \\
\hline Normal & $22(59,5 \%)$ & $17(58,6 \%)$ & Referencia \\
\hline Sobrepeso & $7(18,9 \%)$ & $4(13,8 \%)$ & $0,73(0,18$ a 2,94$)$ \\
\hline Obesidad leve & $1(2,7 \%)$ & $3(10,3 \%)$ & $3,88(0,37$ a 40,70$)$ \\
\hline Obesidad mórbida & $0(0 \%)$ & $0(0 \%)$ & - \\
\hline Datos faltantes & $3(8,1 \%)$ & $2(6,9 \%)$ & - \\
\hline
\end{tabular}


Tabla 2. Descripción de los modelos de regresión logística múltiple utilizados.

Modelos

Modelo 1

Modelo 2

Modelo 3

Tabla 3. Odds ratios multivariados de las variables estadísticamente significativas en los modelos de regresión logística utilizados.

\begin{tabular}{ccc} 
Modelo & Variable & OR (IC95\%) \\
Modelo 1 & Contacto con paciente TB MDR o XDR & $14,56(1,52$ a 139,54) \\
Modelo 2 & Ninguna variable resulto estadísticamente significativa & - \\
Modelo 3 & Edad & $0,94(0,90$ a 0,99$)$ \\
\hline
\end{tabular}

contacto TB resultaron no significativas. En el estudio realizado en un distrito de Lima, Perú, se encontró que tener un contacto intradomiciliario con TB era un factor de riesgo estadísticamente significativo ${ }^{(11)}$. En el presente estudio no observamos una asociación con esta variable, pero sí con el hecho de tener específicamente un contacto TB MDR o TB XDR. Esto podría tener una explicación si consideramos la posibilidad de que las formas de tuberculosis sensible y resistente tengan una distribución diferente en Lima y en Callao ${ }^{(16)}$. Según la información preliminar que muestra el Ministerio de Salud, en Lima existe un mayor porcentaje de pacientes con TB MDR del total de pacientes con TB a comparación de Callao. Por tanto, en Callao se necesitaría hacer un estudio que incluya más pacientes para poder detectar el efecto que teóricamente tiene la variable contacto intradomiciliario TB. Una situación diferente sucede con la variable contacto TB MDR o TB $\mathrm{XDR}$, donde probablemente la asociación teórica que tiene esta con el hecho de tener TB MDR primaria es más directa que la que tiene tener cualquier forma de TB (sensible o resistente) con presentar TB MDR primaria; en el primer caso, se necesitaría una menor cantidad de pacientes que en el segundo para detectar el efecto de la variable independiente.

En el presente estudio hemos observado que una edad mayor a 40 años fue un factor protector significativo en uno de los modelos de análisis multivariado. Este hallazgo concuerda con el Estudio Nacional de Vigilancia de la Resistencia a Drogas Antituberculosas, que refiere que la mayoría de los casos de TB MDR se da en personas jóvenes, entre 15 y 24 años ${ }^{(17)}$.

Entre las limitaciones de nuestro estudio se encuentra el haber utilizado fuentes de información secundarias. Por otro lado, el número de sujetos estudiados fue pequeño.

En conclusión, se ha encontrado que el factor de riesgo para TB MDR primaria fue tener contacto con pacientes TB MDR/XDR; y una edad menor a 40 años fue un factor protector para contraer TB MDR primaria en pacientes del Callao.

1. MINSA - ESNPyCTB: Estrategia Sanitaria Nacional de Prevención y Control de la Tuberculosis. Sala situacional TB 2012. Disponible en: http://www. tuberculosis.minsa.gob.pe/.

2. Del Castillo H, Mendoza A, Saravia J, Somocurcio J. Epidemia de tuberculosis multidrogo resistente y extensivamente resistente a drogas (TB MDR/ XDR) en el Perú: situación y propuestas para su control. Rev peru med exp salud publica. 2009;26(3):380-6.

3. Bonilla C. Situación de la tuberculosis en el Perú. Acta méd peruana. 2008;25(3):163-70.

4. WHO/IUATLD. Anti-tuberculosis drug resistance in the world. Global Project on Anti-Tuberculosis Drug Resistance Surveillance 1994-1997. Geneva: World Health Organization, 1998. Disponible en: http://whqlibdoc.who.int/hq/1997/

\section{REFERENCIAS BIBLIOGRÁFICAS} WHO_TB_97.229.pdf.
5. Trébucq A, Anagonou S, Gninafon M, Lambregts $\mathrm{K}$, Boulahbal F. Prevalence of primary and acquired resistance of Mycobacterium tuberculosis to antituberculosis drugs in Benin after 12 years of short-course chemotherapy. Int J Tuberc Lung Dis. 1999;3(6):466-70.

6. World Health Organization. Global Tuberculosis Report. Annex 4: Global, regional and countryspecific data for key indicators.WHO, 2012. Disponible en: http://www.who.int/tb/publications/ global_report/gtbr12_annex4.pdf.

7. Mathuria JP, Samaria JK, Srivastava GN, Mathuria BL, Ojha SK, Anupurba S. Primary and acquired drug resistance patterns of Mycobacterium tuberculosis isolates in India: a multicenter study. J Infect Public Health. 2013;6(6):456-64. doi: 10.1016/j. jiph.2013.03.003.

8. Zhdanova S, Heysell SK, Ogarkov O, Boyarinova G, Alexeeva G, Pholwat S, et al. Primary multidrugresistant Mycobacterium tuberculosis in 2 regions, Eastern Siberia, Russian Federation. Emerg Infect Dis. 2013;19(10):1649-52. doi: 10.3201/ eid1910.121108.

9. World Health Organization. Guidelines for surveillance of drug resistance in tuberculosis: Fourth Edition. Geneva: WHO, 2003. Disponible en: http://www.sld.cu/galerias/pdf/sitios/tuberculosis/ vig_res_oms.pdf.

10. Suchindran S, Brouwer ES, Van Rie A. Is HIV infection a risk factor for multi-drug resistant tuberculosis? A systematic review. PLoS One. 2009;4(5):e5561.

11. Shah L, Choi H, Krapp F, Zamudio C, Seas C, Ciampi A, et al. Predictors of primary multiple drug resistant tuberculosis (MDR-TB) transmission in Lima, Peru. Int J Infect Dis. 2010;14(1):e25-e26.

12. Yoshiyama T, Supawitkul S, Kunyanone N, Riengthong D, Yanai $\mathrm{H}$, et al. Prevalence of drug-resistant tuberculosis in an HIV endemic area in northern Thailand. Int J Tuberc Lung Dis. 2001;5(1):32-9.

13. Schwoebel V, Decludt B, de Benoist AC, Haeghebaert S, Torrea G, et al. Multidrug resistant tuberculosis in France 1992-4: two case-control studies. BMJ. 1998;317(7159):630-1.

14. Conaty SJ, Hayward AC, Story A, Glynn JR, Drobniewski FA, et al. Explaining risk factors for drug-resistant tuberculosis in England and Wales: contribution of primary and secondary drug resistance. Epidemiol Infect. 2004;132(6):1099-108.

15. Joseph P, Severe P, Ferdinand S, Goh KS, Sola C, et al. Multidrug-resistant tuberculosis at an HIV testing center in Haiti. Aids. 2006;20(3):415-8.

16. Ministerio de Salud. Estadistica. Indicadores Trazadores: TBC. 2014. Disponible en: http://www.app. minsa.gob.pe/bsc/Detalle_IndBSC.asp?lcind=19 \&lcobj=4\&lcper=1\&lcfreg=31/1/2014.

17. Asencios L, Quispe N, Mendoza A, Leo E, Vásquez L, Jave O y col. Vigilancia Nacional de la Resistencia a Medicamentos Antituberculosos, Perú 2005-2006. Rev peru med exp salud publica. 2009;26(3):278-87.

Artículo recibido el 4 de febrero de 2014 y aceptado para publicación el 12 de abril de 2014.

Los autores declaran no tener conflictos de interés.

Correspondencia:

Ana Cecilia Avalos-Rodríguez

Correo electrónico: avalos.ana31@gmail.com 